\title{
A Non-Intrusive Algorithm for Sensitivity Analysis of Chaotic Flow Simulations
}

\author{
Patrick J. Blonigan* \\ NASA Ames Research Center, Moffett Field, CA, 94035, USA \\ Qiqi Wang ${ }^{\dagger}$ \\ Massachusetts Institute of Technology, Cambridge, MA, 02139, USA \\ Eric J. Nielsen ${ }^{\ddagger}$ \\ NASA Langley Research Center, Hampton, VA, 23681 \\ Boris Diskin ${ }^{\S}$ \\ National Institute of Aerospace, Hampton, VA, 23666
}

\begin{abstract}
We demonstrate a novel algorithm for computing the sensitivity of statistics in chaotic flow simulations to parameter perturbations. The algorithm is non-intrusive but requires exposing an interface. Based on the principle of shadowing in dynamical systems, this algorithm is designed to reduce the effect of the sampling error in computing sensitivity of statistics in chaotic simulations. We compare the effectiveness of this method to that of the conventional finite difference method.
\end{abstract}

\section{Nomenclature}

1

$a$

A

B

$C_{D}$

$C_{L}$

$D$

D

R

$r, s, b$

$t$

$m \times 1$ vectors of ones

Speed of sound

$m \times 1$ vector of homogeneous tangent solution weights

$m \times 1$ vector with projection of tangent onto homogeneous tangent solutions

Drag coefficient

Lift coefficient

Drag

Design variable of interest

Objective functions or instantaneous contribution to objective functions

Objective function sensitivities

$n \times 1$ linearization of instantaneous contribution to objective function

Time dilation instantaneous contribution to sensitivity of objective function

Number of time segments

$$
\text { Lift }
$$

$m \times m$ identity matrix

Mach number

Number of homogeneous tangent solutions

Number of states (degrees of freedom)

$n \times 1$ vector of conserved variables

$n \times 1$ spatial residual vector

*Postdoctoral Fellow, NPP/USRA, NASA Ames Research Center, Moffett Field, CA

${ }^{\dagger}$ Associate Professor, Aeronautics and Astronautics, Cambridge, MA, AIAA Member.

${ }^{\ddagger}$ Research Scientist, Computational AeroSciences Branch, MS 128, AIAA Associate Fellow.

$\S$ NIA Research Fellow, AIAA Associate Fellow. 


$\begin{array}{ll}T & \text { Time-averaging window length } \\ U & \text { Fluid velocity } \\ \mathbf{V} & n \times m \text { tangent instantaneous solution matrix } \\ x, y, z & \text { Lorenz system states } \\ \Delta t & \text { Time step } \\ \epsilon & \text { Finite difference perturbation } \\ \rho & \text { Fluid density } \\ \mathbf{\Phi} & \text { Map to state at time } t \\ W & \text { Objective-function windowing weight }\end{array}$

\author{
Subscript \\ $j \quad j$ th column of a matrix \\ $\infty \quad$ Freestream value \\ Superscript \\ $F \quad$ Final state or objective in a time segment \\ I Initial state or objective in a time segment \\ $i \quad$ Time segment \\ - $\quad$ Indicates time-averaged quantity
}

\title{
I. Introduction
}

Many important phenomena in aerospace engineering applications exhibit chaotic dynamics. Examples of chaotic simulations include high-fidelity, scale-resolving turbulence simulations, ${ }^{1}$ such as direct numerical simulations (DNS), large eddy simulations (LES), or detached eddy simulations (DES). Aerospace applications including jet engines, ${ }^{2}$ scramjet engine combustors, ${ }^{3}$ and complex launch vehicle configurations ${ }^{4}$ need to be analyzed with DES or LES to capture key physical phenomena such as flow separation. Multidisciplinary simulations, such as coupled fluid-structure simulations, can also exhibit chaotic dynamics. ${ }^{5-7}$

Design optimization requires computing the sensitivity of quantities of interest with respect to design parameters. Such sensitivity analysis is particularly challenging when the quantity of interest is an infinitetime-average, also called a statistic, of quasi-equilibirium chaotic dynamics. One example of such a quantity of interest is the long-time-averaged root-mean-square (RMS) norm of pressure on a launch vehicle. ${ }^{4} \mathrm{~A}$ finite-time-average approximation of any statistic, necessarily incurs a sampling error, ${ }^{8}$

$$
e_{T}=c T^{-0.5}
$$

where $T$ is the time averaging window size and $c$ is some constant. This sampling error decays with the square root of the averaging window and makes the conventional finite-difference method very noisy for most practical unsteady simulations.

To illustrate the impact of the sampling error on sensitivities computed with finite-differences, we use the Lorenz attractor, a simple chaotic system with three degrees of freedom

$$
\begin{aligned}
& \frac{d x}{d t}-s(y-x)=0, \\
& \frac{d y}{d t}-x(r-z)+y=0, \\
& \frac{d z}{d t}-x y+b z=0 .
\end{aligned}
$$

The sensitivity of the time averaged objective

$$
\frac{1}{T} \int_{T_{0}}^{T_{0}+T}(z-28)^{2} d t
$$

with respect to the Rayleigh number $r$ is computed. Figure 1 (a) plots the objective function, averaged over $T=5$ time units following $T_{0}=5$ time units of run up time. Note that the longest time scale of the 


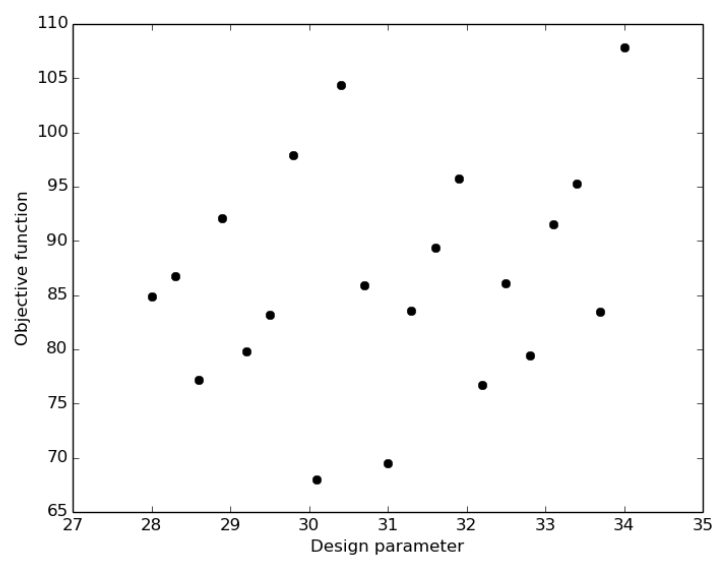

(a) $T=5$

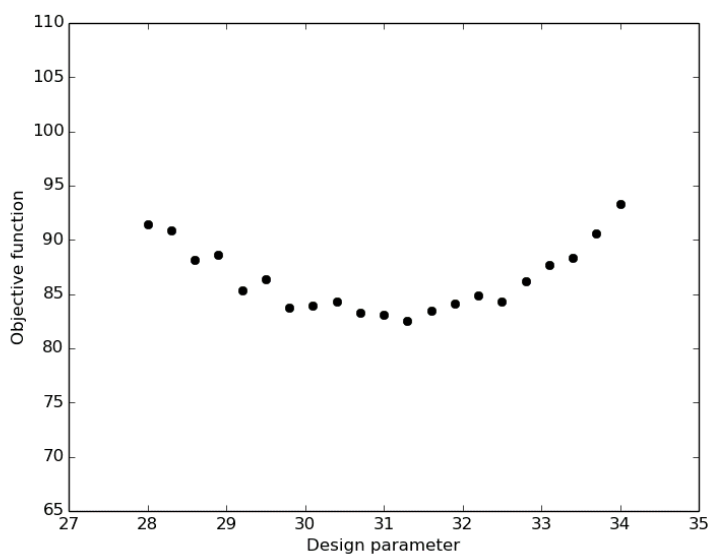

(c) $T=500$

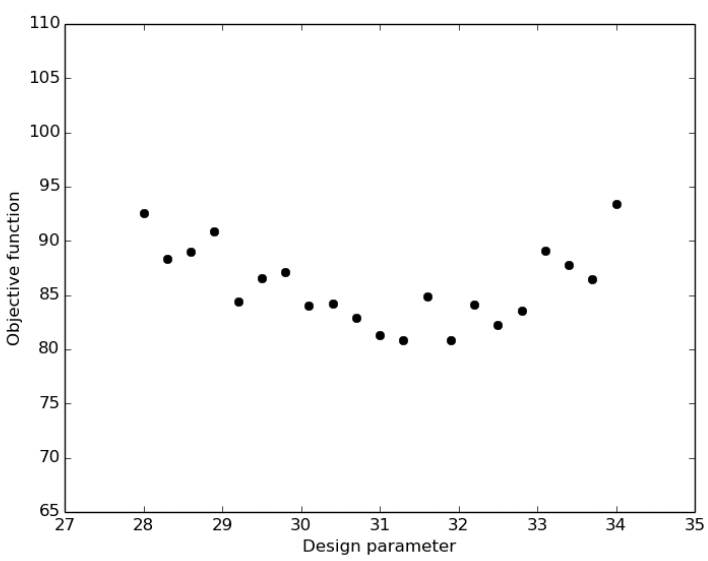

(b) $T=50$

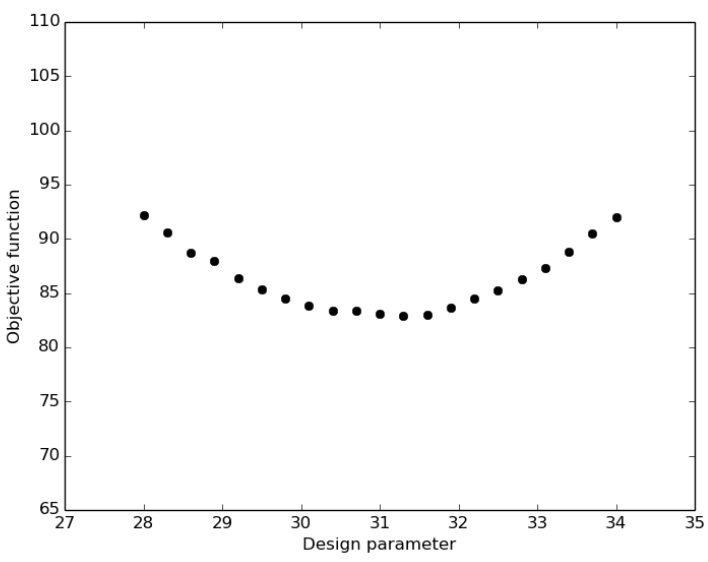

(d) $T=5,000$

Figure 1: Time average of $(z-28)^{2}$ in the Lorenz attractor (Eq. (2)) over four different time spans $T$, as a function of $r$, for $b=8 / 3$ and $s=10$.

dynamics of the Lorenz attractor is about 1 time unit. Despite being averaged over a time span several times longer than the longest time scale of the system, the computed statistic shows no trend. The noise due to sampling error is so large that it is impossible to infer how the objective function depends on the parameter.

To reduce the sampling error, one can increase the averaging length, as shown in Figures 1 (b), (c), and (d). These figures indicate that there is a clear minimum of the statistic when $r \approx 31$. From Eq. (1), reducing sampling error by a factor of 10 would require increasing the window size by a factor of 100 . For high-fidelity simulations, the computation time becomes infeasible fairly quickly.

The least-squares shadowing (LSS) method $^{9}$ has been developed to address this challenge in sensitivity analysis. The original LSS algorithm was intrusive, requiring significant modifications to any nonlinear solver, including development of an appropriate strong linear solver. A less intrusive algorithm has recently been developed. ${ }^{10}$ This paper demonstrates its utility on a chaotic, eddy-resolving turbulent flow simulation.

\section{The Non-Intrusive Shadowing Algorithm}

The algorithm presented in this paper is a modification of the non-intrusive Least Squares Shadowing (NI-LSS) algorithm ${ }^{10}$ that aims to further reduce the intrusiveness. In particular, the algorithm presented in this paper requires neither a tangent (linearized) solver nor an adjoint solver, approximating the tangent 
algorithm presented by $\mathrm{Ni}$ et al. ${ }^{10}$ with finite differences.

We assume that we have a simulator that solves an $n$-dimensional autonomous ODE,

$$
\frac{\partial \mathbf{Q}}{\partial t}+\mathbf{R}(\mathbf{Q}, \mathbf{D})=0
$$

for a given initial state, $\mathbf{Q}^{I}$, a given parameter $\mathbf{D}$, and a given time $t$. The simulator would compute the solution state after the ODE evolves for time $t$. We also assume that the simulator computes a number of quantities of interest, $\mathbf{F}(\mathbf{Q}, \mathbf{D})$, averaged over the time span $t$. Lastly, we also need it to compute these quantities of interest at the end of the time integration.

Mathematically, the simulator can be represented as three maps:

1. $\mathbf{\Phi}(\mathbf{Q}, \mathbf{D}, t)$ gives the solution state after a time $t$ evolution of the ODE at parameter $\mathbf{D}$, starting from initial state $\mathbf{Q}$.

2. $\overline{\mathbf{F}}(\mathbf{Q}, \mathbf{D}, t)$ gives the time averaged $\mathbf{F}(\mathbf{Q}, \mathbf{D})$ over a time $t$ evolution of the ODE at parameter $\mathbf{D}$, starting from initial state $\mathbf{Q}$.

3. $\mathbf{F}^{E}(\mathbf{Q}, \mathbf{D}, t)$ gives $\mathbf{F}(\mathbf{\Phi}(\mathbf{Q}, \mathbf{D}, t), \mathbf{D})$, the instantaneous value of the quantities of interest at the end of the evolution.

The NI-LSS algorithm runs this simulation to perform sensitivity analysis of the long time averaged objective $\mathbf{F}$,

$$
\mathbf{f}:=\lim _{T \rightarrow \infty} \frac{1}{T} \int_{0}^{T} \mathbf{F}(\mathbf{Q}, \mathbf{D}) d t
$$

with respect to $\mathbf{D}$. It does this by computing $m+1$ finite-difference approximated tangent solutions in $K$ size $T / K$ time segments, then solving a size $m n \times m n$ least squares system. The algorithm proceeds as follows:

1. Choose an integer $m$, an estimate of the number of unstable modes of the ODE. Choose a time span $\Delta t$, an estimate to the reciprocal of the largest Lyapunov exponent. ${ }^{11}$ Then choose the number of time spans $T / \Delta t$, such that $T$ is a multiple of the largest time scale in the system.

2. Run the simulation for sufficient time to a quasi-equilibrium-state. Denote the state as $\mathbf{Q}^{0}$. From a dynamical systems point of view, this means that $\mathbf{Q}^{0}$ can be considered as being on the attractor of the system.

3. Set $\mathbf{V}^{0}$ to a random matrix of dimension $(n, m)$ where $n$ is the number of degrees of freedom of the dynamical system. Set $\hat{\mathbf{V}}^{0}$ to a zero vector of dimension $n$. Start from time segment $i=1$.

4. Compute $\mathbf{R}\left(\mathbf{Q}^{i-1}, \mathbf{D}\right)$. This can be obtained either directly from the simulator, or approximating it with $-\left(\mathbf{\Phi}\left(\mathbf{Q}^{i-1}, \mathbf{D}, \delta t\right)-\mathbf{Q}^{i-1}\right) / \delta t \approx-\frac{d \mathbf{Q}}{d t}$ for a small $\delta t$.

5. Project each column of $\mathbf{V}^{i-1}$ as well as $\hat{\mathbf{V}}^{i-1}$ against $\mathbf{R}$, i.e.,

$$
\mathbf{V}^{i-1}=\mathbf{V}^{i-1}-\frac{\mathbf{R} \mathbf{R}^{T} \mathbf{V}^{i-1}}{\mathbf{R}^{T} \mathbf{R}}, \quad \hat{\mathbf{V}}^{i-1}=\hat{\mathbf{V}}^{i-1}-\frac{\mathbf{R} \mathbf{R}^{T} \hat{\mathbf{V}}^{i-1}}{\mathbf{R}^{T} \mathbf{R}},
$$

6. Run the simulator to compute

$$
\mathbf{Q}^{i}=\mathbf{\Phi}\left(\mathbf{Q}^{i-1}, \mathbf{D}, \Delta t\right), \quad \overline{\mathbf{F}}^{i}=\overline{\mathbf{F}}\left(\mathbf{Q}^{i-1}, \mathbf{D}, \Delta t\right), \quad \mathbf{F}^{E, i}=\mathbf{F}^{E}\left(\mathbf{Q}^{i-1}, \mathbf{D}, \Delta t\right),
$$

7. For $j=1, \ldots, m$, denote $\mathbf{V}_{j}^{i}$ as the $j$ th column of the $n \times m$ matrix $\mathbf{V}^{i}$. Run the simulator to compute

$$
\mathbf{V}_{j}^{i}=\frac{\boldsymbol{\Phi}\left(\mathbf{Q}^{i-1}+\epsilon \mathbf{V}_{j}^{i-1}, \mathbf{D}, \Delta t\right)-\mathbf{Q}^{i}}{\epsilon}, \quad \mathbf{G}_{i}=\frac{\overline{\mathbf{F}}\left(\mathbf{Q}^{i-1}+\epsilon \mathbf{V}_{j}^{i-1}, \mathbf{D}, \Delta t\right)-\overline{\mathbf{F}}^{i}}{\epsilon}, \quad H_{i}=\frac{\mathbf{R}^{T} \mathbf{V}^{i-1}}{\Delta t \mathbf{R}^{T} \mathbf{R}},
$$

8. Run the simulator to compute

$$
\begin{gathered}
\hat{\mathbf{V}}^{i}=\frac{\boldsymbol{\Phi}\left(u_{i-1}+\epsilon \hat{\mathbf{V}}^{i-1}, \mathbf{D}+\epsilon, \Delta t\right)-\mathbf{Q}^{i}}{\epsilon}, \quad \hat{\mathbf{G}}^{i}=\frac{\overline{\mathbf{F}}\left(\mathbf{Q}^{i-1}+\epsilon \hat{\mathbf{V}}^{i-1}, \mathbf{D}+\epsilon, \Delta t\right)-\overline{\mathbf{F}}^{i}}{\epsilon}, \quad \hat{H}_{i}=\frac{\mathbf{R}^{T} \hat{\mathbf{V}}^{i-1}}{\Delta t \mathbf{R}^{T} \mathbf{R}}, \\
4 \text { of } 8
\end{gathered}
$$


9. Compute the QR decomposition

$$
\mathbf{V}^{i}=\mathcal{Q R}^{i}
$$

then compute

$$
\mathbf{B}^{i}=\mathcal{Q}^{T} \hat{\mathbf{V}}^{i}
$$

and set $V_{i}=\mathcal{Q}, \hat{\mathbf{V}}^{i}=\hat{\mathbf{V}}^{i}-\mathcal{Q} \mathbf{B}^{i}$.

10. Loop back to Step 4 with $i=i+1$, until $i>T / \Delta t=K$.

11. Solve the reduced Least Squares Shadowing system

$$
\min \left\|\begin{array}{c}
\mathbf{A}^{1} \\
\vdots \\
\mathbf{A}^{K} \\
\mathbf{A}^{K+1}
\end{array}\right\|_{2} \text { s.t. } \quad\left[\begin{array}{llll}
-\mathcal{R}^{1} & \mathbf{I} & & \\
& \ddots & \ddots & \\
& & -\mathcal{R}^{K} & \mathbf{I}
\end{array}\right]\left[\begin{array}{c}
\mathbf{A}^{1} \\
\vdots \\
\mathbf{A}^{K} \\
\mathbf{A}^{K+1}
\end{array}\right]=\left[\begin{array}{c}
\mathbf{B}^{1} \\
\vdots \\
\mathbf{B}^{K}
\end{array}\right]
$$

compute the sensitivity

$$
\frac{d \mathbf{f}}{d D} \approx \sum_{i=1}^{K} w^{i}\left(\left(\mathbf{G}^{i}+H^{i} \mathbf{1}\right) \mathbf{A}^{i}+\left(\hat{\mathbf{G}}^{i}+\hat{H}^{i}\right)\right),
$$

where $w_{i}$ is an averaging weight series satisfying $\sum_{i=1}^{K} w^{i}=1$. In this paper,

$$
w^{i}=\frac{\left(\sin \frac{i \pi}{K+1}\right)^{2}}{K+1} .
$$

This algorithm is implemented in the open source repository https://github.com/qiqi/fds . All the results presented in this paper are based on this implementation of the algorithm.

\section{Two-Dimensional Chaotic Airfoil}

To demonstrate the NI-LSS algorithm, we consider a NACA 0012 airfoil at an angle-of-attack of $\alpha=20^{\circ}$ with a freestream Mach number $M_{\infty}=0.1$ and a Reynolds number of 10,000. This flow is similar to the chaotic vortex shedding flows previously studied by Pulliam. ${ }^{12}$ The flow was simulated using FUN3D's compressible, second order, finite volume flow solver.

\section{A. Sensitivity Estimates}

To demonstrate the NI-LSS algorithm, we consider the sensitivity of time-averaged lift and drag to perturbations of the freestream Mach number, $M_{\infty}$, and the angle of attack, $\alpha$. The Mach number sensitivities can be estimated analytically by assuming that $d C_{L} / d M_{\infty}=d C_{D} / d M_{\infty}=0$. This is a reasonable assumption for the small Mach number, $M_{\infty}=0.1$, considered in this paper. Since the flow is nearly incompressible the effect of Mach number on $C_{L}$ and $C_{D}$ is $\mathcal{O}\left(M^{2}\right) .{ }^{14}$

If $d C_{L} / d M_{\infty}=0$, then for a unit reference area

$$
L=\frac{1}{2} \rho_{\infty} U_{\infty}^{2} C_{L}=\frac{1}{2} \rho_{\infty} a_{\infty}^{2} M_{\infty}^{2} C_{L}
$$

Since the freestream density $\rho_{\infty}$ and speed of sound $a_{\infty}$ are constants

$$
\frac{d L}{d M_{\infty}}=\rho_{\infty} a_{\infty}^{2} M_{\infty} C_{L}=2 \frac{L}{M_{\infty}}
$$

By the same reasoning,

$$
\frac{d D}{d M_{\infty}}=\rho_{\infty} a_{\infty}^{2} M_{\infty} C_{D}=2 \frac{D}{M_{\infty}}
$$

Figure 2, shows that the analytical estimates are quite accurate. 


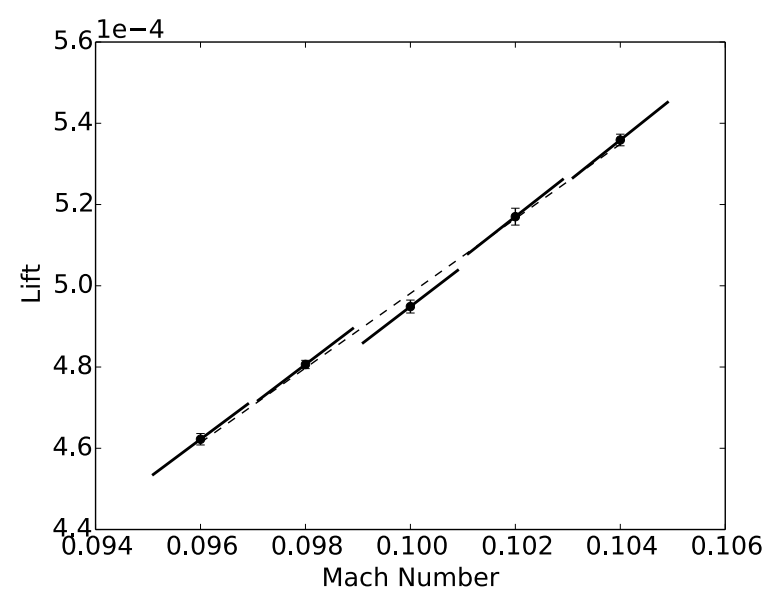

(a) Lift

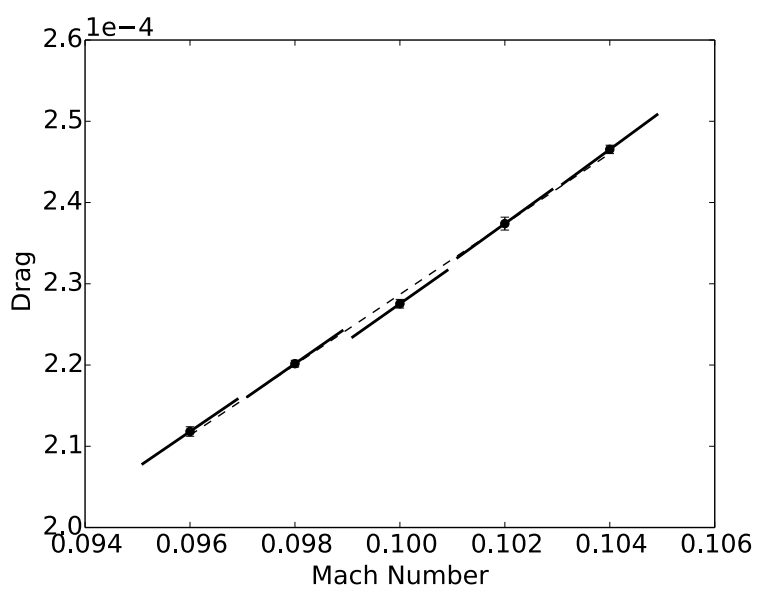

(b) Drag

Figure 2: Time-averaged lift and drag versus Mach number. The dotted line is a linear regression, the solid lines show local gradients computed with Eqs. (4) and (5). All cases were run for 500,000 time steps and averaged for 490,000 steps. Error bars are estimated using the batch mean approach with four means. ${ }^{13}$

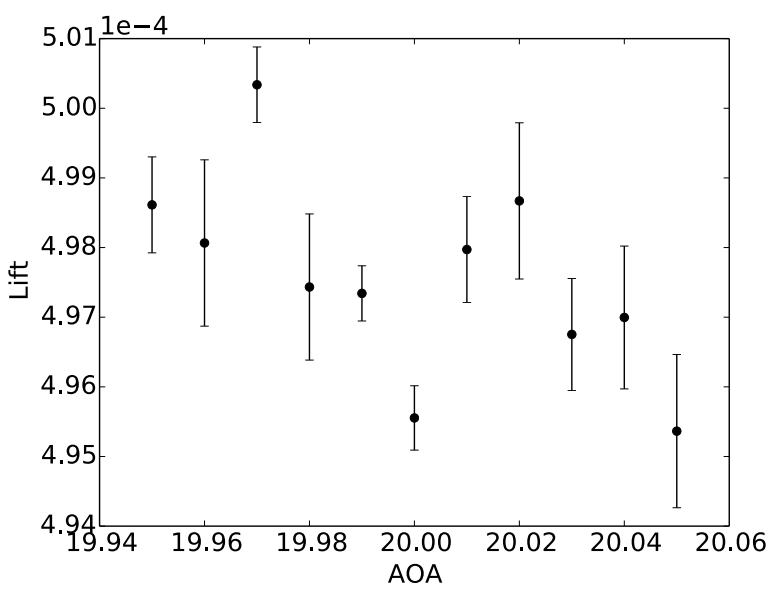

(a) Lift

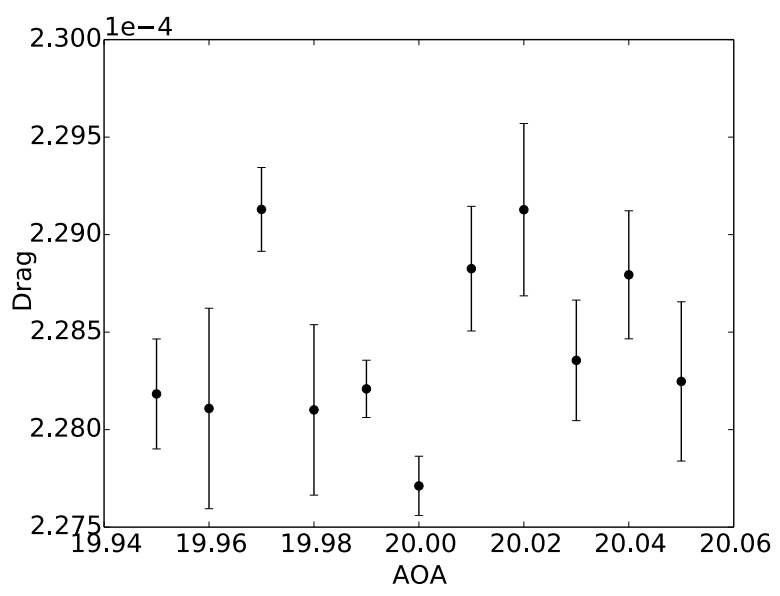

(b) Drag

Figure 3: Time-averaged lift and drag versus angle of attack. All cases were run for 1,010,000 time steps and averaged for 1,000,000 steps. Error bars are estimated using the batch mean approach with four means. ${ }^{13}$

We do not have similar analytical estimates for the sensitivities to the angle of attack. In an attempt to numerically estimate the sensitivities, we performed 11 simulations in the range of $\alpha \in[19.95,20.05]$, each for 1,010,000 time steps. The lift and drag were averaged over the last 1,000,000 time steps, corresponding to 100,000 non-dimensional time units (non-dimensionalized with respect to the freestream speed of sound and the chord length), or 10,000 flow-through times (non-dimensionalized with respect to the freestream velocity and the chord length). The time-averaged lift and drag are shown in Figure 3. In spite of these lengthy calculations, no definite trend can be inferred.

\section{B. LSS Sensitivity Results}

The NI-LSS algorithm has been applied to estimate sensitivities with respect to the Mach number and the angle of attack. One hundred time segments are used in each calculation. Each time segment contains 200 


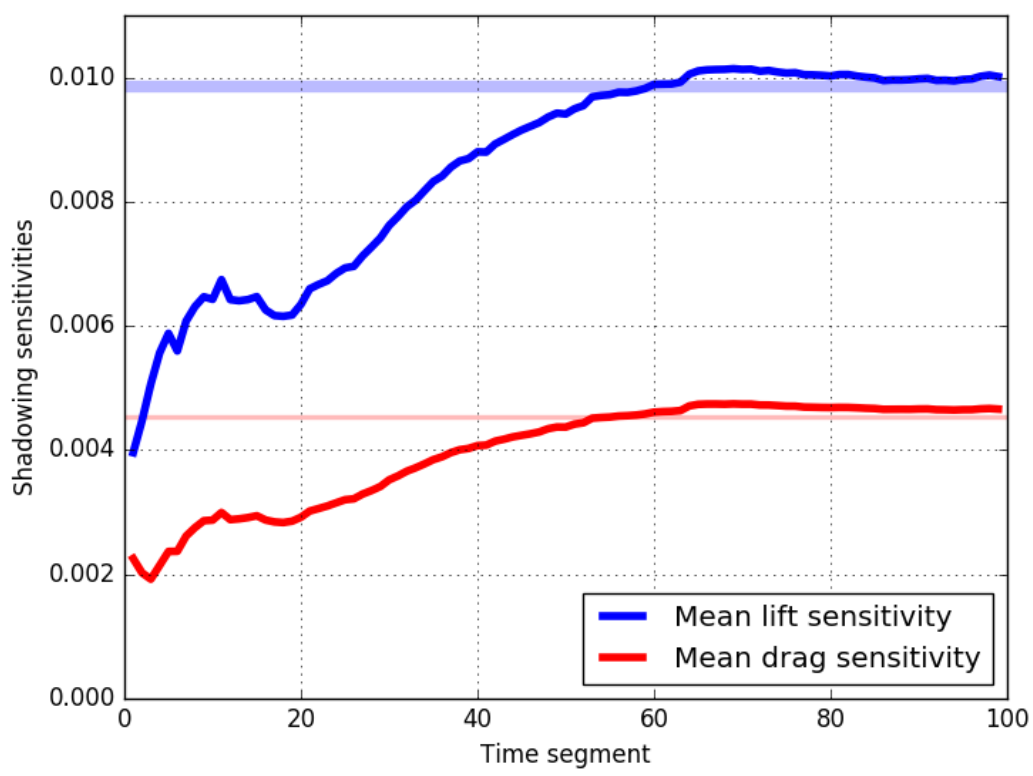

Figure 4: Shadowing sensitivity for the derivative of lift and drag to the Mach number. The x-axis indicates the number of time segments, each containing 200 time steps, used in the shadowing sensitivity analysis. The light-colored bands indicate the analytical estimates.

time steps, which corresponds to 20 non-dimensional time units (non-dimensionalized with respect to the freestream speed of sound and the chord length), or two flow-throughs (non-dimensionalized with respect to the freestream velocity and the chord length). In the calculation, we used $m=16$ homogeneous tangent solutions, which requires $m+2=18$ simulations for each time segment. The total computation involves $18 \times 100 \times 200=360,000$ time steps. The sensitivities are computed after each time segment is completed. Figure 4 shows the lift and drag sensitivities with respect to the Mach number versus the number of time segments. Figure 5 shows corresponding sensitivities with respect to the angle of attack.

In Figure 4, we can see that the NI-LSS sensitivities approach the analytical estimates as the number of time segments increases. However, we do not observe a similar convergence for the sensitivity with respect to the angle of attack. We think that the lack of convergence arises because the sensitivities with respect to the angle of attack are small; and as a result, neither finite difference (as shown in Figure 3) nor the current NI-LSS algorithm (as shown in Figure 5) can accurately compute such small sensitivities.

\section{Conclusion}

We presented a modified non-intrusive least-squares shadowing (NI-LSS) algorithm for computing the sensitivity of statistics in chaotic flow simulations. The algorithm minimizes modifications of the flow solver. The NI-LSS algorithm is applied to a two-dimensional simulation of a stalled NACA 0012 airfoil at Reynolds number 10,000, performed using the FUN3D flow solver. Sensitivities of drag and lift with respect to the Mach number and the angle of attack have been computed and compared to finite-difference estimates. Mach number sensitivities have also been compared with analytical estimates. The NI-LSS sensitivities with respect to Mach number agree with finite-difference and analytical estimates. Neither NI-LSS nor the conventional finite-difference approach is able to estimate the sensitivities with respect to the angle of attack.

\section{Acknowledgments}

Funding for Patrick Blonigan was provided by the NASA Postdoctoral Program administered by the Universities Space Research Association (USRA). 


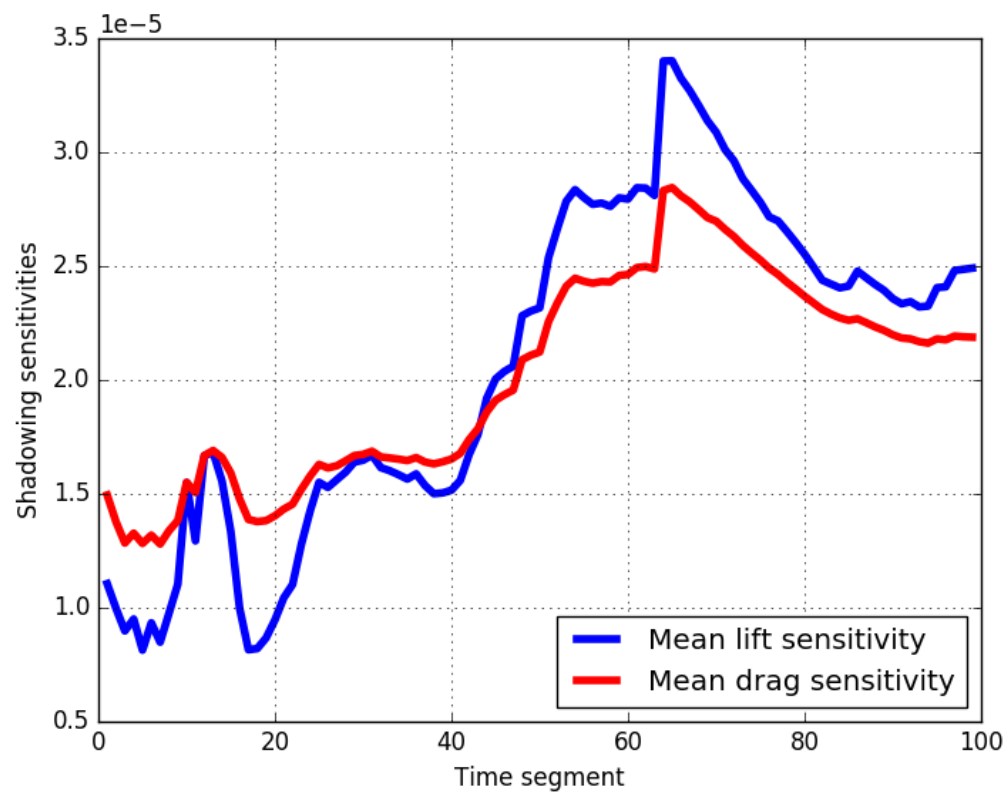

Figure 5: Shadowing sensitivity for the derivative of lift and drag to the angle of attack. The x-axis indicates the number of time segments, each containing 200 time steps, used in the shadowing sensitivity analysis.

\section{References}

${ }^{1}$ Keefe, L., Moin, P., and Kim, J., "The dimension of attractors underlying periodic turbulent Poiseuille flow," Journal of Fluid Mechanics, Vol. 242, 1992, pp. 1-29.

${ }^{2}$ Leonard, T., Gicquel, L., Gourdain, N., and Duchaine, F., "Steady/Unsteady Reynolds-Averaged Navier-Stokes and Large Eddy Simulations of a Turbine Blade at High Subsonic Outlet Mach Number," Journal of Turbomachinery, Vol. 137, Apr 2015, pp. 041001-1.

${ }^{3}$ Larsson, J., Laurence, S. J., Bermejo-Moreno, I., Bodart, J., Karl, S., and Vicquelin, R., "Incipient thermal choking and stable shock-train formation in the heat-release region of a scramjet combustor. Part II: Large eddy simulations," Combustion and Flame, Vol. 162, 2015, pp. 907-920.

${ }^{4}$ Alter, S. J., Brauckmann, G. J., Kleb, B., Glass, C. E., Streett, C. L., and Schuster, D. M., "Time-Accurate Unsteady Pressure Loads Simulated for the Space Launch System at Wind Tunnel Conditions," AIAA 2015-3149.

${ }^{5}$ Dowell, E., "Nonlinear oscillations of a fluttering plate," AIAA Journal, Vol. 4, No. 7, 1966, pp. 1267-1275.

${ }^{6}$ Ostoich, C. M., Bodony, D. J., and Geubelle, P. H., "Interaction of a Mach 2.25 turbulent boundary layer with a fluttering panel using direct numerical simulation," Physics of Fluids, Vol. 25, 2013, pp. 110806.

${ }^{7}$ Lee, B., Price, S., and Wong, Y., "Nonlinear aeroelastic analysis of airfoils: bifurcation and chaos," Progress in Aerospace Sciences, Vol. 35, No. 3, 1999, pp. 205-334.

${ }^{8}$ Zwanzig, R. and Ailawadi, N. K., "Statistical Error Due to Finite Time Averaging in Computer Experiments," Physical Review, Vol. 182, No. 1, 1969, pp. 280.

${ }^{9}$ Wang, Q., Hu, R., and Blonigan, P., "Least Squares Shadowing sensitivity analysis of chaotic limit cycle oscillations," Journal of Computational Physics, Vol. 267, 2014, pp. 210-224.

${ }^{10} \mathrm{Ni}$, A., Blonigan, P. J., Chater, M., Wang, Q., and Zhang, Z., "Sensitivity analysis on chaotic dynamical system by Non-Intrusive Least Square Shadowing (NI-LSS)," AIAA 2016-4399.

${ }^{11}$ Benettin, G., Galgani, L., Giorgilli, A., and Strelcyn, J., "Lyapunov characteristic exponents for smooth dynamical systems and for Hamiltonian systems; a method for computing all of them. Part 2: Numerical application," Meccanica, Vol. 15, No. 1, 1980, pp. 21-30.

${ }^{12}$ Pulliam, T., "Low Reynolds number numerical solutions of chaotic flow," AIAA-1989-123.

${ }^{13}$ Fishman, G. S., "Grouping Observations in Digital Simulation," Management Science, Vol. 24, No. 5, 1978 , pp. 510-521.

${ }^{14}$ Anderson, J. D., Modern compressible flow: with historical perspective, Vol. 12, McGraw-Hill New York, 1990. 